\title{
Spin state as a probe of vesicle self-assembly
}

\author{
Sanghoon Kim, Christine Bellouard*, Julian Eastoe, Nadia Canilho, Sarah E Rogers, Dris \\ Ihiawakrim, Ovidiu Ersen and Andreea Pasc*
}

\section{Experimental section}

\section{Chemicals}

Dodecyltrimethylammonium bromide (DTAB, $\geq 98 \%$ ), sodium bis(2-ethylhexyl) sulfosuccinate (NaAOT, 98\%), iron (III) nitrate anhydride $\left(\mathrm{Fe}\left(\mathrm{NO}_{3}\right)_{3}\right)$, and Deuterium oxide $\left(\mathrm{D}_{2} \mathrm{O}\right)$ were purchased from Sigma-Aldrich. Iron (III) chloride $\left(\mathrm{FeCl}_{3}\right)$ was purchased from Alfa Aesar. Water was deionized and purified using a Milli-Q pack system. All reagents were used without further purification.

\section{Synthesis of magnetic surfactant $\mathrm{AOT}_{3} \mathrm{~F}\left(\left(\mathrm{C}_{20} \mathrm{H}_{37} \mathrm{O}_{7} \mathrm{~S}\right)_{3} \mathrm{Fe}\right)$}

$\mathrm{AOT}_{3} \mathrm{~F}$ was synthesized as reported previously with slight modification. ${ }^{1} 250 \mathrm{~mL}$ of a saturated aqueous solution of the iron (III) nitrate was filtered and added to $125 \mathrm{~mL}$ of $1.0 \mathrm{~mol} \mathrm{~L}^{-1}$ of NaAOT (sodium bis(2-ethylhexyl) sulfosuccinate) solution prepared in absolute ethanol. $50 \mathrm{~mL}$ of diethyl ether was added to the mixture, and then the lower aqueous phase containing excess of iron (III) nitrate was removed. The organic solvent was evaporated using a rotary evaporator and then, the surfactant $\mathrm{AOT}_{3} \mathrm{~F}$ was dried in vacuo at $60^{\circ} \mathrm{C}$ for $24 \mathrm{~h}$.

\section{Synthesis of magnetic surfactant DTAF $\left(\mathrm{CH}_{3}\left(\mathrm{CH}_{2}\right)_{11} \mathrm{~N}\left(\mathrm{CH}_{3}\right)_{3} \mathrm{FeCl}_{3} \mathrm{Br}\right)$}

DTAF was synthesized as reported previously. ${ }^{2}$ Briefly, $1.63 \mathrm{~g}$ of $\mathrm{FeCl}_{3}(10.1 \mathrm{mmol})$ was added to $3.10 \mathrm{~g}$ of DTAB $(11.0 \mathrm{mmol})$ that was already dissolved in $50 \mathrm{~mL}$ of methanol. Then, the solution was heated to reflux overnight. The methanol was evaporated using a rotary evaporator and then, the surfactant DTAF was dried in vaccuo at $60^{\circ} \mathrm{C}$ for $24 \mathrm{~h}$. 


\section{Preparation of magnetic vesicles}

Magnetic vesicle dispersions were prepared by mixing the magnetic surfactants in $\mathrm{D}_{2} \mathrm{O}$ at room temperature under vortex stirring, if necessary. Briefly, $23.9 \mathrm{mg}$ of $\mathrm{AOT}_{3} \mathrm{~F}$ and $31.8 \mathrm{mg}$ of DTAF were dissolved in $4 \mathrm{~mL}$ of $\mathrm{D}_{2} \mathrm{O}$ and this system was denoted as A-series (molar ratio of $\mathrm{DTAF} / \mathrm{AOT}_{3} \mathrm{~F}$ is 3.7). Another vesicle dispersion was prepared by dissolving $11.2 \mathrm{mg}$ of $\mathrm{AOT}_{3} \mathrm{~F}$ and $31.7 \mathrm{mg}$ of DTAF in $5 \mathrm{~mL}$ of $\mathrm{D}_{2} \mathrm{O}$, which is denoted as B-series (molar ration of $\mathrm{DTAF} / \mathrm{AOT}_{3} \mathrm{~F}$ is 8.1 ). Vesicle dispersions were kept at $25^{\circ} \mathrm{C}$ for $2 \mathrm{~h}$ before further analysis.

\section{Cryogenic transmission electron microscopy and scanning transmission electron microscopy.}

Preparation and analysis of the samples by Cryo-TEM and STEM: A drop of the sample was deposited on an electron microscopy grid covered by a hydrophobic carbon membrane. The drop size was progressively reduced in order to obtain a thin film covering the whole membrane. The grid was subsequently plunged into liquid ethane at liquid nitrogen temperature. By maintaining the specimen at this temperature, the grid was transferred on the cryo-holder and inserted in the high-vacuum column of the electron microscope. The presence of the thin ethane layer covering the samples provides an additional protection against radiation damage. The specimens have been analyzed on a JEOL 2100F (S)TEM microscope working at $200 \mathrm{kV}$ and equipped by a probe aberration corrector, an EELS (Gatan Tridiem) spectrometer and an EDX (Si-Li) detector. This set-up allows to reach resolutions of $2 \AA$ and $1.1 \AA$ under TEM and STEM modes, respectively. For limiting irradiation damage, the images were acquired by using a low density of the electron beam irradiation.

\section{Dynamic light scattering and nanoparticle tracking analysis.}

Particle sizing by Dynamic light scattering (DLS) was obtained using a Malvern 3000HSA Zetasizer. The Contin algorithm that takes into account the polydispersity of the sample has been used. Nanoparticle tracking analysis (NTA) was performed with a Malvern NS300 Nanosight. In case of nanoparticle tracking analysis, vesicle solutions were diluted with water in situ inside the Nanosight instrument. NTA was configured to work with a $405 \mathrm{~nm}$ laser and a $430 \mathrm{~nm}$ long-pass filter. Surface tensions were measured on the Krüss-K100 tensiometer at $25^{\circ} \mathrm{C}$ using the plate method. 


\section{Small-Angle Neutron Scattering (SANS)}

Scattering was measured on the LOQ and SANS2D diffractometers at the ISIS Facility, Rutherford Appleton Laboratory, United Kingdom. LOQ and SANS2D are both time-of-flight instruments. SANS2D uses neutrons with an incident wavelength of $2.2 \AA<\lambda<14 \AA$, and with the $1 \mathrm{~m}^{2}$ detector offset sideways and vertically by $150 \mathrm{~mm}$, the resulting q range was $0.006 \AA^{-1}$ $<\mathrm{q}<0.7 \AA^{-1}$ For LOQ the incident wavelengths were $2.2 \AA<\lambda<10 \AA$, giving $0.006 \AA^{-1}<\mathrm{q}<$ $0.24 \AA^{-1}$. The raw scattering data were corrected for the detector efficiencies, sample transmission, and background scattering and converted to scattering cross-section data $(\delta \Sigma / \delta \Omega$ vs Q) using instrument-specific software Mantid. ${ }^{3,4}$ SANS data were placed on an absolute scale $\left(\mathrm{cm}^{-1}\right)$ using the scattering from a standard sample (Water in Hellma fused silica cuvettes with a path length of $2 \mathrm{~mm}$ ) in accordance with established procedures at ISIS. ${ }^{5}$

\section{SANS. Scattering Laws and Model Fitting}

Scattered intensity I(q) is a product of the size and shape of the aggregates (form factor, $\mathrm{P}(\mathrm{q})$ ) and the interaction between these aggregates (structure factor, $\mathrm{S}(\mathrm{q})$ ),

$$
I(q) \propto P(q, R) S(q), \quad \text { where } \mathrm{R} \text { is the particle radius. }
$$

The SANS from vesicle dispersions were fitted using the program Sasview using the VesicleModel. $^{6}$

The VesicleModel provides the form factor, $\mathrm{P}(\mathrm{q})$, for an unilamellar vesicle. The form factor is normalized by the volume of the shell. ${ }^{7,8}$

The 1D scattering intensity is calculated in the following way:

$$
P(q)=\frac{\text { scale }}{V_{\text {shell }}}\left[\frac{3 V_{1}\left(\rho_{1}-\rho_{2}\right) J_{1}\left(q R_{1}\right)}{q R_{1}}+\frac{3 V_{2}\left(\rho_{2}-\rho_{\text {solv }}\right) J_{1}\left(q R_{2}\right)}{q R_{2}}\right]^{2}+b k g
$$

where scale is a scale factor, $V_{\text {shell }}$ is the volume of the shell, $V_{1}$ is the volume of the core, $V_{2}$ is the total volume, $R_{1}$ is the radius of the core, $R_{2}$ is the outer radius of the shell, $\rho_{1}$ is the scattering length density of the core and the solvent, $\rho_{2}$ is the scattering length density of the shell, and bkg is the background level, and $\left.J_{1}=(\sin x-x \cos x) / x^{2}\right)$. The functional form is 
identical to a "typical" core-shell structure, except that the scattering is normalized by the volume that is contributing to the scattering, namely the volume of the shell alone. Also, the vesicle is best defined in terms of a core radius $\left(=\mathrm{R}_{1}\right)$ and a shell thickness, $\mathrm{t}$.

Herein, the adjustable parameters in the model are thickness $(t)$ and core radius of the shell $\left(\mathrm{R}_{1}\right)$.

\section{Magnetic measurements}

Magnetic measurements have been performed with a Physical Properties Measurements System (PPMS) from Quantum Design equipped with ACMS option, and using a sample holder provided for liquids. All measurements have been made with the same heavy water solutions as for SANS. The signal contribution of sample holder and heavy water have been measured in the same conditions as the solutions, it is purely diamagnetic and has been systematically subtracted. A remaining diamagnetic contribution arising from the surfactants is taken into account in the fitting procedure. All samples have been introduced directly at $60 \mathrm{~K}$ in the sample chamber, and temperature measurements have been performed between $60 \mathrm{~K}$ and $2 \mathrm{~K}$ under several applied fields with decreasing and increasing temperature at $0.5 \mathrm{~K} / \mathrm{min}$. No discrepancies have been observed between both measurements for A- and B-series.

\section{Results}

Transmission electron microscopy and scanning transmission electron microscopy.

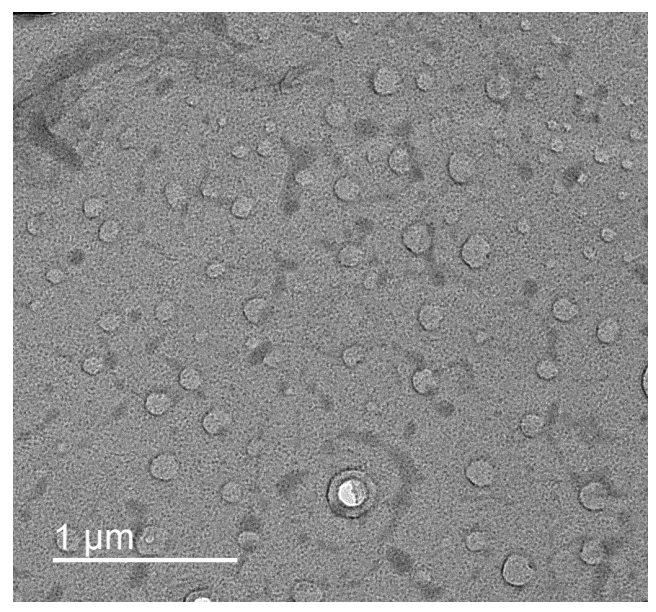

Figure S1: Cryo-TEM images of vesicles observed for system B2. 


\section{Dynamic light scattering.}

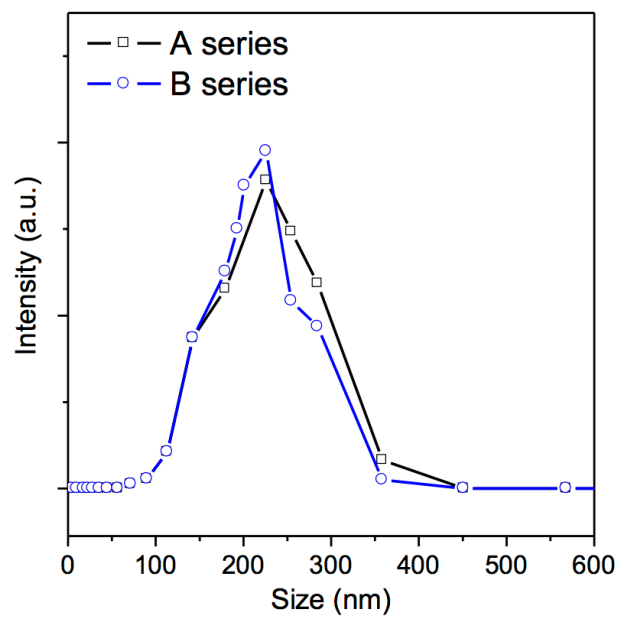

Figure S2. Size distribution of MagSurf vesicles measured for systems A1 and B1, using DLS.

Figure S2 which gathers DLS results for A1 and B1 solutions shows that the size distribution of vesicles are superimposed.

\section{Critical aggregation concentration (CAC) of DTAF/AOT3F in A and B series}

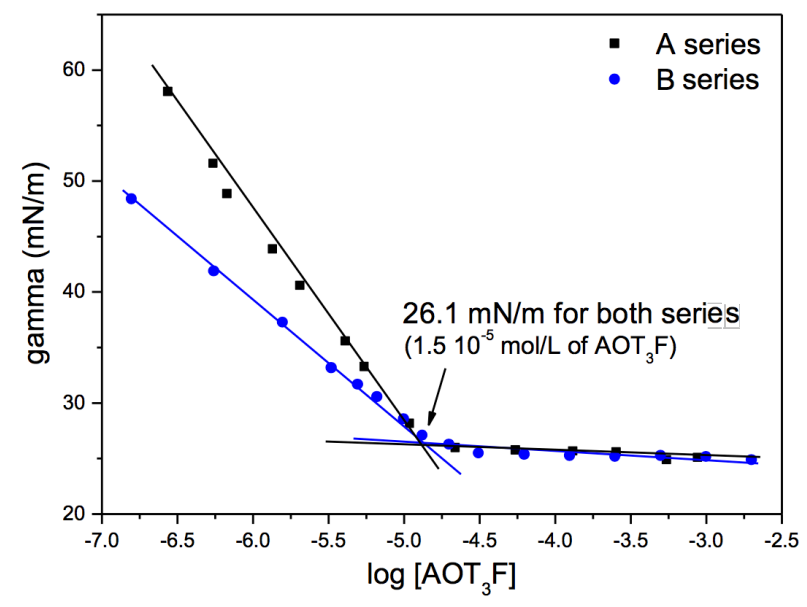

Figure S3. Critical aggregation concentration (CAC) of vesicle systems determined using Surface tension measurements. Surface tension $(\mathrm{mN} / \mathrm{m})$ is plotted as a function of $\log$ of concentration $(\mathrm{M})$ of $\mathrm{AOT}_{3} \mathrm{~F}$ in the A series $(\mathrm{r}=3.7)$ and $\mathrm{B}$ series $(\mathrm{r}=8.1)$. 


\section{Fitting procedure of magnetization measurements:}

The low field $(\mathrm{H}=5 \mathrm{kOe})$ temperature magnetization has been fitted with the contribution of two Brillouin functions as :

$$
M(H, T)=f_{5 / 2} M_{s}^{5 / 2} B_{5 / 2}(H, T-\theta)+\left(1-f_{5 / 2}\right) M_{s}^{1 / 2} B_{1 / 2}(H, T)+d(H)
$$

$f_{5 / 2}$ is the fraction of spin $5 / 2, M_{S}^{x}$ is the saturation magnetization of spin $\mathrm{x}, B_{x}(H, T)$ is the Brillouin function, for $\mathrm{S}=5 / 2, \theta$ is introduced to take into account spin-spin interactions. $d(H)$ corresponds the diamagnetic contribution which is temperature independent. The measured magnetization has been corrected for this contribution and plotted in Figure 4. An additional spin crossover at low temperature $(\leq 5 \mathrm{~K})$ is indeed present, as clearly observed in the high field measurements for sample B4 (Figure 4b). As a consequence, to obtain reliable parameters, fitting has been limited to the $4 \mathrm{~K}-60 \mathrm{~K}$ temperature range for $\mathrm{Ax}$ and $\mathrm{B} 1-2$, and to $5 \mathrm{~K}-60 \mathrm{~K}$ for $\mathrm{B} 3-4$. The fitting parameters, $f_{5 / 2}$ and $\theta$, are reported in Table $\mathrm{S} 1$.

\section{Parameters fitted to SANS and magnetic data}

\begin{tabular}{|c|c|c|c|c|c|c|}
\hline Sample & $\mathrm{r}^{\mathrm{a}}$ & $\mathrm{C}_{\mathrm{T}}^{\mathrm{b}}$ & $\begin{array}{l}\text { Diameter } \\
\text { (nm) }\end{array}$ & $\begin{array}{l}\text { Shell thickness } \\
(\mathrm{nm})\end{array}$ & $\begin{array}{l}\text { High spin } \\
\text { fraction, } f_{5 / 2}\end{array}$ & $\begin{array}{c}\theta \\
(\mathrm{K})\end{array}$ \\
\hline A1 & 3.7 & 21.4 & 183 & 2.5 & 90 & -1.1 \\
\hline $\mathrm{A} 2$ & 3.7 & 10.7 & 182 & 2.4 & 87 & -1.0 \\
\hline A3 & 3.7 & 5.35 & 175 & 2.6 & 89 & -1.2 \\
\hline A4 & 3.7 & 2.68 & - & - & 67 & -0.9 \\
\hline B1 & 8.1 & 15.2 & 177 & 2.4 & 60 & -1.4 \\
\hline B2 & 8.1 & 7.58 & 180 & 2.4 & 60 & -1.2 \\
\hline B3 & 8.1 & 3.78 & - & - & 40 & $-0.2 \pm 0.1$ \\
\hline B4 & 8.1 & 1.89 & - & - & 23 & 0 \\
\hline
\end{tabular}

Table S1. Parameters used to fit SANS and magnetization data of solutions; $a$ : $r$ is the molar concentration ratio $\mathrm{DTAF} / \mathrm{AOT}_{3} \mathrm{~F} ; \mathrm{b}: \mathrm{C}_{\mathrm{T}}$ is the sum of the surfactant molar concentration expressed in $\mathrm{mM}$.

The $\theta$ value is close to $-1 \mathrm{~K}$ for the Ax series, which reveals low antiferromagnetic interactions between $\mathrm{Fe}^{3+}$ spins which could arise from dipolar interactions. $\theta$ is then a function of number 
and distance between $\mathrm{Fe}^{3+}{ }_{5 / 2}$ neighbors. In fact $\theta$ is almost identical for all systems studied. The A series reveals that the local order around $\mathrm{Fe}^{3+}$ with $\mathrm{S}=5 / 2$ is similar in vesicles $(\mathrm{A} 1-3)$ and in membranes (A4).

\section{High spin fraction of single surfactant solutions.}

The magnetic behavior of single surfactant systems have been investigated and analyzed under the same conditions as the A and B samples.

Heavy water DTAF solutions have been prepared at three concentrations: one above the CMC concentration $(29.5 \mathrm{mM})$, and two below $(5.90 \mathrm{mM}$ and $1.48 \mathrm{mM})$. Figure $\mathrm{S} 4$ presents the magnetizations measured as a function of temperature for two applied fields.

The temperature dependence between $2 \mathrm{~K}$ and $60 \mathrm{~K}$, under $5 \mathrm{kOe}$, for the concentration far above the $\mathrm{CMC}$, can be satisfactorily fitted with the same formula as A and B solutions. This provides the proportion of high spins of $97 \%$ with $\theta=-3 \mathrm{~K}$. This feature can be obviously attributed to $\mathrm{Fe}^{3+}$ spins involved in micelles, the high absolute value of $\theta(3 \mathrm{~K})$ compared to the value obtained with vesicles $(1 \mathrm{~K})$ can be readily related to the fact that the distances between $\mathrm{Fe}^{3+}$ nearest neighbors is lower for pure DTAF molecular assembly structures than in $\mathrm{AOT}_{3} \mathrm{~F} / \mathrm{DTAF}$ complexes.

For the concentration far below the CMC $(1.48 \mathrm{mM})$, which corresponds to a large proportion of monomers, the signal is reduced, indicating a much lower proportion of high spins. Moreover, a spin crossover is observed at $10 \mathrm{~K}$, with an applied field of $70 \mathrm{kOe}$. From a fit of the magnetization measured under $5 \mathrm{kOe}$, and for $\mathrm{T}>10 \mathrm{~K}$, the low spin fraction can be evaluated to about $60 \%$. At low temperature, the magnetization measured under $70 \mathrm{kOe}$ is close to the saturation magnetization of $\mathrm{Fe}^{3+}$ with $\mathrm{S}=1 / 2$ (100 emu / gFe). These features support the statement that low spin value can be associated to monomers.

For an intermediate concentration $(5.9 \mathrm{mM})$, below the $\mathrm{CMC}$, the signal is only slightly lower than in case of micelles $(29.5 \mathrm{mM})$. Within the assumption that high spins belong to DTAF assembled structures, these results support the hypothesis of premicellar aggregation and a progressive and continuous process for micelle formation. ${ }^{9}$ 


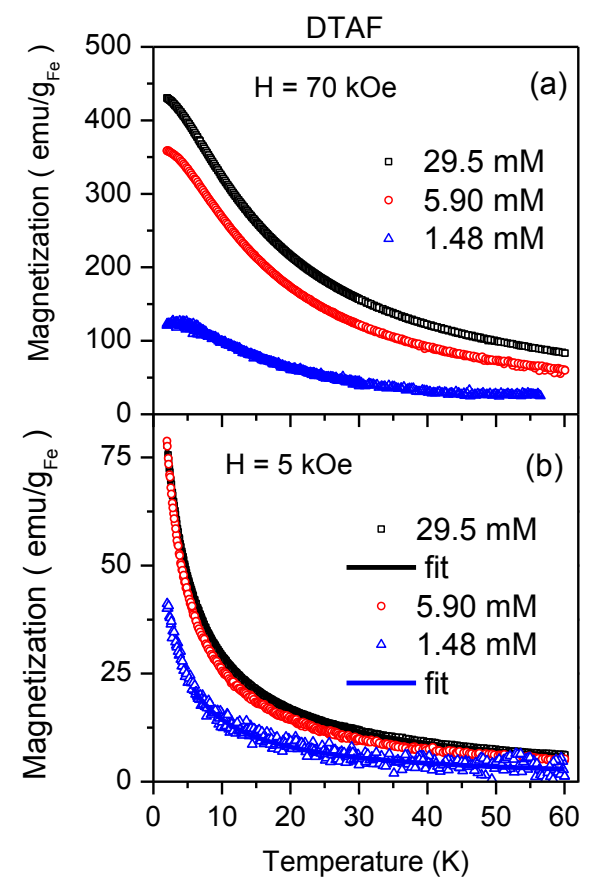

Figure S4: Magnetization as a function of temperature for 3 DTAF solutions, above the CMC: $29.5 \mathrm{mM}$ and below the CMC: 5.90 and $1.48 \mathrm{mM}$. (a): $\mathrm{H}=70 \mathrm{kOe},(\mathrm{b}): \mathrm{H}=5 \mathrm{kOe}$.

Heavy water $\mathrm{AOT}_{3} \mathrm{~F}$ solutions, below and above the critical micelle concentration, have also been prepared to investigate the magnetic behavior of pure $\mathrm{AOT}_{3} \mathrm{~F}$. Figure S5 shows that, whatever the concentration, the signal remains low (maximum at $200 \mathrm{emu} / \mathrm{g}$ ), far below the expected signal of a $5 / 2$ spin (saturation at $500 \mathrm{emu} / \mathrm{g}$ ). For the concentration $(0.50 \mathrm{mM})$ below the $\mathrm{CMC}$, the high spin fraction deduced from a fit (for $\mathrm{T}>10 \mathrm{~K}$, and $\mathrm{H}=10 \mathrm{kOe}$ ) can be estimated at $0.30 \pm 0.05$. A clear spin crossover is observed around $T=5 \mathrm{~K}$ with a maximum in the magnetization as a function of temperature for both applied fields (10 kOe and $70 \mathrm{kOe})$, which was also observed for DTAF below the CMC at $70 \mathrm{kOe}$.

The presence of monomers, or even micelles of $\mathrm{AOT}_{3} \mathrm{~F}$, can be associated with a dominating contribution of $1 / 2$ spins. The fact that the iron centers remain in a low spin state even in micelles is quite intriguing, and is not in line with observations on related systems composed either from DTAF, or mixtures of DTAF and $\mathrm{AOT}_{3} \mathrm{~F}$. This can be explained if in $\mathrm{AOT}_{3} \mathrm{~F}$ micelles, the iron centers are more exposed to water as compared to DTAF. This may be related to the presence of three bulky AOT moieties around $\mathrm{Fe}^{3+}$. 


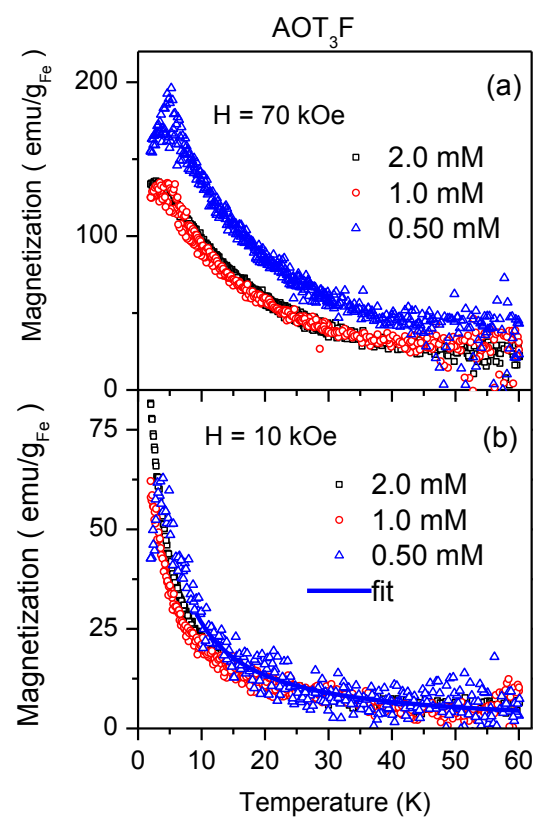

Figure S5: Magnetization as a function of temperature for three $\mathrm{AOT}_{3} \mathrm{~F}$ solutions, below and above the CMC: (a): $\mathrm{H}=70 \mathrm{kOe},(\mathrm{b}): \mathrm{H}=10 \mathrm{kOe}$.

In conclusion, below the $\mathrm{CMC}$, both surfactants exhibit a paramagnetic response in the temperature range $10 \mathrm{~K}-60 \mathrm{~K}$ dominated by $1 / 2$ spins, which can then be attributed to monomers.

\section{References}

(1) Eastoe, J.; Fragneto, G.; Robinson, B. H.; Towey, T. F.; Heenan, R. K.; Leng, F. J. J. Chem. Soc., Faraday Trans. 1992, 88, 461.

(2) Brown, P.; Bushmelev, A.; Butts, C. P.; Cheng, J.; Eastoe, J.; Grillo, I.; Heenan, R. K.; Schmidt, A. M. Angew. Chem. Int. Ed. 2012, 51, 2414.

(3) Heenan, R. K.; Penfold, J.; King, S. M. J. Appl. Crystallogr. 1997, 30, 1140.

(4) http://www.mantidproject.org

(5) Heenan, R. K.; Rogers, S. E.; Turner, D.; Terry, A. E.; Treadgold, J.; King, S. M. Neutron News 2011, 22, 19.

(6) http://www.sasview.org/index.html

(7) Guinier, A.; Fournet, G. Small-Angle Scattering of X-Rays; John Wiley and Sons: New York, 1955. 
(8) Kline, S. R. J. Appl. Cryst. 2006, 39, 895.

(9) Cui, X.; Mao, S.; Liu, M.; Yuan, H.; Du, Y. Langmuir 2008, 24, 10771. 